\title{
Numerical Weather Predictions for GPS Positioning
}

\author{
A.B.O. Jensen \\ Informatics and Mathematical Modelling, Technical University of Denmark, \\ Richard Petersens Plads, Building 321, DK-2800 Lyngby, Denmark
}

\begin{abstract}
When GPS satellite signals are transmitted through the atmosphere they are affected by the media. In the neutral atmosphere the refraction is a function of pressure, temperature, and humidity along the signal path, and in the GPS positioning process this effect is normally handled by utilising global tropospheric delay models. For high accuracy differential positioning over baselines lengths where the differential effect of the signal delay from the neutral atmosphere is significant, these global models of the signal delay are not sufficiently accurate, and this is especially the case during abnormal weather conditions.

This paper describes a new approach where numerical weather predictions (NWPs) are introduced in the GPS data processing instead of global tropospheric delay models. NWPs are predictions of the meteorological conditions for a given area and epoch in time, and can as such be used for estimating the tropospheric delay for a satellite signal by numerical integration along the signal path through the NWP. For the tests described in this paper, the signal delays are determined as a zenith delay through the NWP combined with a mapping function. This approach is useful for kinematic and shorter static GPS applications. The paper describes the theory of the method, and the applicability of the method is evaluated by analysing position accuracies obtained by introducing NWP-derived signal delays in kinematic and static processing of GPS data. Improved position accuracies are obtained for most of the test scenarios, indicating that the method does have a potential.
\end{abstract}

Keywords. GPS positioning, numerical weather predictions (NWP), tropospheric delay

\section{Introduction}

The GPS satellite signals are affected while being transmitted through the lowest parts of the Earth's atmosphere, the ionosphere and the neutral atmosphere. Normally, global atmospheric models are used to correct for the atmospheric effect, and the models are sufficiently accurate for most GPS positioning with modest accuracy demands. For high accuracy differential carrier phase-based positioning the global models are, however, not sufficiently accurate and other measures must be introduced in the data processing in order to handle the atmospheric effects.

The double differencing technique is commonly used since the technique results in cancellation of satellite and receiver clock errors during the processing. When utilising both atmospheric modelling and double differencing, the residual atmospheric effect is considerably mitigated, and position accuracies of a few $\mathrm{cm}$ can be obtained when the distance between the reference and roving GPS receivers is less than approximately $20 \mathrm{~km}$ [4].

For longer GPS vectors the ionospheric error can be further mitigated using dual frequency equipment and the so called ionosphere free linear combinations of the L1 and L2 observations [16]. With this technique the first order effects of the ionosphere are removed from the data processing, but also the noise level is increased by generating the linear combinations. For satellite signals received at high elevation angles the size of the residual higher order ionospheric effects and the increased noise are negligible [8], but for signals received at elevation angles below 15 degrees, the effects become significant and should be taken into consideration during the data processing, especially if the GPS vectors are longer than 50-100 $\mathrm{km}$ [3].

The effect caused by the neutral atmosphere, normally referred to as the tropospheric delay, is handled by using global tropospheric delay models. When the ionosphere free linear combination is used in the GPS data processing, the residual tropospheric effect after both modelling and double differencing is the dominant error source for carrier phase-based positioning with distances ranging from $20 \mathrm{~km}$ to a few hundred $\mathrm{km}$ between reference and rover. This is the case under normal conditions when the ionospheric activity is low or moderate. During high ionospheric activity there might be significant higher order ionospheric effects even though the ionosphere free linear combination has been used. 
For most geodetic and geodynamic GPS applications the positioning is based on long observation time spans, and the considerable amount of data available leaves enough degrees of freedom in the adjustment process to estimate the residual tropospheric effect as a separate element in the adjustment. This approach is used for instance by the Bernese software [1].

For other applications, such as Real Time Kinematic (RTK) positioning or kinematic post processed positioning there is normally not enough data, from each position solution, to actually estimate the size of the residual tropospheric error. Therefore, other methods for dealing with the residual tropospheric effect must be used. In the following a new approach for estimating the tropospheric delay is described.

\section{The tropospheric delay}

The tropospheric delay is caused by refraction of the satellite signal in the lowest parts of the Earth's atmosphere, extending from the surface of the Earth up to an altitude of approximately $50 \mathrm{~km}$. This part of the atmosphere, covering the troposphere and the stratosphere, is referred to as the neutral atmosphere by meteorologists since it is electrically neutral to radio waves.

Refraction in the neutral atmosphere causes a signal delay which is normally referred to as the tropospheric delay in GPS terminology, because the majority of the effect occurs in the troposphere. The tropospheric signal delay can be determined by integrating the refractivity $(\mathrm{N})$, along the signal path (ds):

$$
\text { delay }=10^{-6} \int \mathrm{N} \mathrm{ds}
$$

The refractivity is a function of the meteorological parameters; pressure, temperature and humidity, and can be described by Equation 2.2 as given for instance in [9].

$$
\mathrm{N}=\mathrm{k}_{1}\left(\frac{\mathrm{P}_{\mathrm{d}}}{\mathrm{T}}\right) \mathrm{Z}_{\mathrm{d}}^{-1}+\left(\mathrm{k}_{2} \frac{\mathrm{e}}{\mathrm{T}}+\mathrm{k}_{3} \frac{\mathrm{e}}{\mathrm{T}^{3}}\right) \mathrm{Z}_{\mathrm{w}}^{-1}
$$

Where $\mathrm{k}_{1}, \mathrm{k}_{2}$, and $\mathrm{k}_{3}$ are constants, $\mathrm{Pd}$ is the partial pressure of dry air, $\mathrm{T}$ is temperature, and $\mathrm{e}$ is the partial pressure of water vapor. $Z_{d}$ and $Z_{w}$ are the compressibility factors for dry air and water vapor respectively, and they are accounting for the deviation of the gasses from an ideal gas.
By combining Equation 2.1 and 2.2 an expression for the tropospheric signal delay is obtained, and the effect of the signal delay, converted to metric units, is a range error of approximately $2.3-2.5$ meters in zenith for a GPS receiver located at sea level. The size of the error increases for signals received at lower elevation angles.

Normally the tropospheric delay is handled using global tropospheric delay models as, for instance the Hopfield model [5] or the Saastamoinen model [13]. In 1999 V. Mendes performed a comprehensive analysis of global tropospheric delay models, and he found the Saastamoinen model to be one of the best models, with an estimated accuracy of $3 \mathrm{~cm}$ in zenith [10]. For lower elevation angles the model becomes less accurate, indicating the need for either dealing with the residual tropospheric effects in the GPS data processing, or for investigating methods to obtain more accurate estimates of the signal delay. Numerical weather predictions (NWPs) can be used in the second case as described in the following section.

\section{NWPs for tropospheric modelling}

Numerical Weather Predictions (NWPs) are three dimensional models of the atmospheric conditions for a given area and point in time. The models form the basis for weather predictions and contain the necessary information for estimating the refractivity along the path of a GPS signal.

Equation 2.2 can be rewritten to obtain the following expression:

$$
\mathrm{N}=\mathrm{k}_{1} \mathrm{R}_{\mathrm{d}} \rho+\frac{\mathrm{R}_{\mathrm{d}}}{\varepsilon}\left(\mathrm{k}_{2}-\mathrm{k}_{1} \varepsilon+\frac{\mathrm{k}_{3}}{\mathrm{~T}}\right) \mathrm{q} \rho
$$

Where the new variable $R_{d}$ is the gas constant for dry air, $\rho$ is the density of air, $\varepsilon$ is the ratio between the gas constants for dry and wet air, and $\mathrm{q}$ is specific humidity. This expression is more suitable for the test purposes described in this paper, since specific humidity is given directly in the weather models used. Further, it is more convenient to perform the integration with respect to air pressure than with respect to altitude, since pressure levels are normally used as the vertical reference in weather predictions. The expression for the signal delay is therefore rewritten, as shown in [7], and Equation 3.2 for the signal delay in the zenith direction is finally obtained.

$$
\text { ztd }=10 \int_{p_{\text {top }}^{-6}}^{\mathrm{p}_{\text {ant }}} \frac{\mathrm{k}_{1} \mathrm{R}_{\mathrm{d}}}{\mathrm{g}} \mathrm{dp}+10^{-6} \int_{\mathrm{p}_{\text {top }}}^{\mathrm{p}_{\text {ant }}} \frac{\mathrm{R}_{\mathrm{d}}}{\varepsilon}\left(\mathrm{k}_{2}-\varepsilon \mathrm{k}_{1}+\frac{\mathrm{k}_{3}}{\mathrm{~T}}\right) \frac{\mathrm{q}}{\mathrm{g}} \mathrm{dp}
$$


The integration is carried out from the pressure at the top of the neutral atmosphere, $p_{\text {top }}$ down to the pressure level at the GPS antenna, $p_{\text {ant }}$. The new variable in this equation is $\mathrm{g}$ which is gravity.

In the derivation of this Equation 3.2 which was first given by $H$. Vedel in [17], the hydrostatic equation is introduced, thus assuming that the atmosphere is in hydrostatic equilibrium. This is an approximation, which is commonly used for weather modelling, and is expected to be valid for the applications given in this paper.

Based on Equation 3.2 the tropospheric zenith delay can now be derived by extracting pressure, temperature and humidity from a numerical weather prediction. For the following tests the DMIHIRLAM-E model was used, which is described in [14]. This model is grid based with a $0.15^{\circ} \times 0.15^{\circ}$ spacing, 31 vertical layers, and it covers all of Europe. Data from 5. and 14. September 2000 was made available by the Danish Meteorological Institute, and the models were given as one hour predictions.

A routine for estimating tropospheric zenith delay based on Equation 3.2 and the DMIHIRLAM-E data was developed. The values for temperature and humidity were determined by linear interpolation for each intersection point between the signal path and layers in the NWP. Also the meteorological parameters for the location of the GPS antenna were determined from the nearest layers in the DMI-HIRLAM-E model by a linear interpolation / extrapolation. The contribution to the zenith delay from above the weather model was determined using the expression given in [17]. For the k-constants in Equation 3.2 the values given in [2] have been used.

\section{Test of NWP derived zenith delays}

The accuracy of the zenith delays derived using the above procedure was verified by a comparison with GPS-derived zenith delays. Twenty four hours of dual frequency GPS data from 14 permanent GPS reference stations located in Denmark and southern Sweden were used to test the accuracy of the NWP derived zenith delays. The GPS data was processed with the Bernese processing software [1], with precise orbits, and accurate station coordinates given in the same reference frame and time epoch as the satellite orbits. The ionospheric effect was handled by introducing the ionosphere free linear combination of the observations, and any errors arising from multipath and receiver noise is expected to be low, because of the high quality equipment installed at the GPS sites. The sites are equipped with choke ring antennas and geodetic grade dual frequency GPS receivers. Residual errors from the GPS data processing are thus only ocean loading effects, which are not modelled in Bernese, but are expected to be negligible for the current stations. Higher order ionospheric effects are also expected to be small, since the ionospheric activity on the observation days was low.

The differences between the GPS derived zenith delays and delays derived from the DMI-HIRLAME data have a mean of $0.6 \mathrm{~cm}$, a standard deviation of $1.6 \mathrm{~cm}$ and an RMS of $1.7 \mathrm{~cm}$. These results were obtained using data from the 14 sites and 16 instances in time. Similar accuracies of NWP derived zenith delays have been obtained by other groups, as described for instance in [12], [15], and [17]. This indicates that the NWP derived zenith delays are better than the Saastamoinen model, considering its estimated accuracy of $3.0 \mathrm{~cm}$ as given in [10].

The use of an improved estimate of the tropospheric delay can improve the accuracy of GPS positions, if the size of the residual errors of all other error sources in the processing is smaller than the size of the improvement in the tropospheric delay estimate. Static and kinematic positioning tests were therefore performed in order to verify whether an improvement in positioning performance could be obtained.

The positioning tests were carried out using commercial GPS processing software, since the method is expected to have a larger impact on shorter static and kinematic processing scenarios which are often handled with commercial software. As mentioned in the introduction, residual tropospheric modelling effects are normally estimated as a part of the positioning process for long static observation campaigns, where the processing is carried out using more advanced software.

\section{Positioning tests}

The positioning tests were carried out using the GPS data from the 14 permanent GPS stations mentioned above and shown in Figure 1. Two six hours data periods from each day were processed with a 15 second data rate. Precise orbits were introduced, and the ionosphere free linear combination of the observations was used to eliminate the majority of the ionospheric effect. The data was processed using the GPSurvey software version 2.35 from Trimble Navigation. 
Station ONSA was used as reference station, and the resulting GPS vector lengths ranged from 35 to $288 \mathrm{~km}$. The largest height difference between reference and roving receiver was 215 meters.

Before processing the data the new tropospheric correction approach was applied to the RINEX GPS data files. Initially zenith delays were estimated from the DMI-HIRLAM-E data for each GPS position at every full hour where weather data was available. A linear temporal interpolation was then carried out in order to estimate the zenith delay for every data epoch. The Niell mapping function [11] was applied to the zenith delays, to determine the slant tropospheric delay, and finally the slant delay was subtracted from the raw code and phase observations in the GPS data files. Tropospherically corrected RINEX GPS data was hereby generated.

The processing was carried out twice firstly with the tropospherically corrected GPS data and then with the Saastamoinen tropospheric correction enabled in the software. All other parameters were identical. The processing was carried out in both static and kinematic mode.

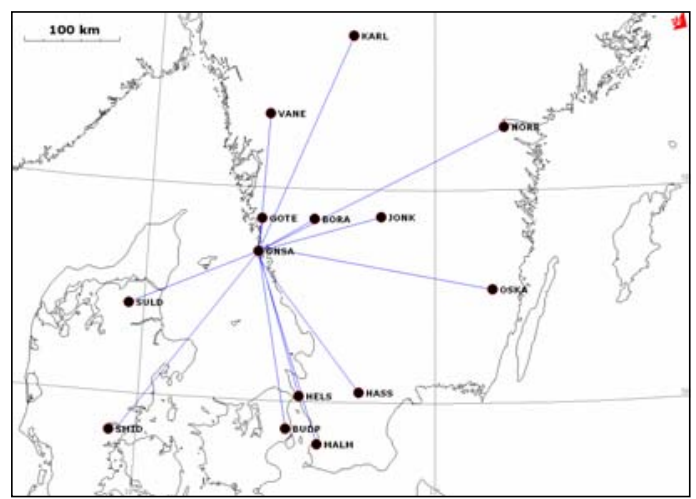

Fig. 1. Location of GPS stations, and vectors used for the positioning tests

\subsection{Static positioning results}

The resulting coordinates from the static processing were compared to the previously known coordinates for the GPS stations. In Table 1 mean and standard deviations are given for the 3D coordinate differences with respect to the previously known stations coordinates, i.e. the size of the vector between the position given by the processing result and the known position. All mean values are therefore positive in the table. It was expected that the effect of using the NWP derived zenith delays would be greater with a larger separation between the reference and roving GPS receivers, or with a larger height difference between the two receivers. This was, however, not the case, and the results of the different static baseline processings are therefore grouped together in the table.

Table 1. Statistics from static positioning test

\begin{tabular}{|c|c|c|}
\hline \multirow[t]{2}{*}{ Static results } & \multicolumn{2}{|c|}{$\begin{array}{c}\text { Mean and standard deviation } \\
\text { of } 3 \mathrm{D} \text { position difference }\end{array}$} \\
\hline & Saastamoinen & NWP approach \\
\hline \multicolumn{3}{|l|}{ 5. Sept. 2000} \\
\hline 00:00-06:00 & $0.135 \pm 0.113 \mathrm{~m}$ & $0.115 \pm 0.068 \mathrm{~m}$ \\
\hline $12: 00-18: 00$ & $0.092 \pm 0.076 \mathrm{~m}$ & $0.086 \pm 0.059 \mathrm{~m}$ \\
\hline \multicolumn{3}{|l|}{ 14. Sept. 2000} \\
\hline $00: 00-06: 00$ & $0.037 \pm 0.032 \mathrm{~m}$ & $0.020 \pm 0.014 \mathrm{~m}$ \\
\hline $12: 00-18: 00$ & $0.052 \pm 0.044 \mathrm{~m}$ & $0.112 \pm 0.070 \mathrm{~m}$ \\
\hline
\end{tabular}

Table 1 shows that both mean and standard deviation are generally smaller when using the NWP approach. The position accuracy was, however, only improved with the NWP approach for 25 of the 51 positions determined, and for the afternoon data set of 14 . September the Saastamoinen model outperforms the NWP approach.

During 14. September a low pressure system was moving over the area creating a relatively high tropospheric activity, and it is expected that this is causing problems with the tropospherically corrected data files during the data preprocessing in GPSurvey. Considering the speed of the GPS satellites and the Niell mapping function, a change in tropospheric slant delay of approximately 0.5 meter occurs when a satellites rises with from a $5.0^{\circ}$ to a $5.1^{\circ}$ elevation angle, if the zenith delay is 2.4 meters [7]. Such a change in elevation angle can take place during 15 seconds, i.e. in the present case from one data epoch to the next. With the tropospherically corrected data files a change in the phase observations of more than two cycles will be interpreted as a cycle slip when the data is imported by GPSurvey. Therefore a $15^{\circ}$ elevation mask was used for the data processing, whereby most of this effect was eliminated for the results given in Table 1. There might, however, have been some remnants of this effect left in the data set collected during the afternoon of 14. September, and it is expected that filtering carried out by GPSurvey, during preprocessing of the data, is accidentally removing parts of the tropospheric corrections. Hereby worse results for the NWP approach are obtained with the afternoon data of 14. September.

The ionospheric activity on the two days was low. But in the afternoon and evening hours of 4 . September, the ionospheric activity was high, and this may have affected the GPS data collected in the 
morning hours of 5. September, resulting in the relatively large mean values given in the second row in Table 1.

\subsection{Kinematic positioning results}

For the kinematic test, the static data sets from the 14 GPS stations were processed in kinematic mode, so one position was determined for each station every 15 second. "On-the-fly" ambiguity resolution was used with the optimal search algorithm implemented in the GPSurvey software. All other processing parameters were the same as for the static processing mentioned above.

The 1440 positions obtained from positioning each of the 13 "roving" GPS receivers through the six hour periods were subtracted from the previously known coordinates of the GPS stations, and the mean and standard deviation of the size of the $3 \mathrm{D}$ error vector, determined as for the static case, are shown in Table 2. Positions determined for time epochs where the RDOP was higher than 4 are not included in the results given in Table 2.

The results of the data from 5. September show an improvement in both mean and standard deviation when using the NWP approach. For the morning of 14. September the results are almost identical for the two tropospheric approaches, but for the afternoon hours the best results are obtained with the Saamstamoinen model. The explanation is expected to be the same as for the static case, i.e. difficulties in GPSurvey with handling large variations in tropospheric delay during data import.

Table 2. Statistics from kinematic positioning test

\begin{tabular}{|c|c|c|}
\hline \multirow[t]{2}{*}{$\begin{array}{l}\text { Kinematic } \\
\text { results }\end{array}$} & \multicolumn{2}{|c|}{$\begin{array}{c}\text { Mean and standard deviation } \\
\text { of } 3 \mathrm{D} \text { position difference }\end{array}$} \\
\hline & Saastamoinen & NWP approach \\
\hline \multicolumn{3}{|l|}{ 5. Sept. 2000} \\
\hline $00: 00-06: 00$ & $0.239 \pm 0.223 \mathrm{~m}$ & $0.231 \pm 0.169 \mathrm{~m}$ \\
\hline 12:00-18:00 & $0.200 \pm 0.122 \mathrm{~m}$ & $0.156 \pm 0.096 \mathrm{~m}$ \\
\hline \multicolumn{3}{|l|}{ 14. Sept. 2000} \\
\hline 00:00-06:00 & $0.098 \pm 0.103 \mathrm{~m}$ & $0.101 \pm 0.102 \mathrm{~m}$ \\
\hline $12: 00-18: 00$ & $0.072 \pm 0.088 \mathrm{~m}$ & $0.144 \pm 0.092 \mathrm{~m}$ \\
\hline
\end{tabular}

Given in Table 3 is the number of positions determined for each of the kinematic processing scenarios.

As shown in Table 3 there is a relatively large difference in the number of positions determined with the two tropospheric approaches. The GPSurvey software does not determine a kinematic position for a given time epoch if the residual noise level in the processing is too high, and the difference in number of positions determined is therefore expected to be caused by differences in the residual noise level. Generally more positions were determined with the NWP approach, and this could be the result of a better modelling of the tropospheric delay, where the amount of unmodelled tropospheric effects is decreased implying a lower noise level in the data processing.

Table 3. Positions determined from kinematic test

\begin{tabular}{|c|c|c|}
\hline \multirow[t]{2}{*}{$\begin{array}{l}\text { Kinematic } \\
\text { results }\end{array}$} & \multicolumn{2}{|c|}{$\begin{array}{c}\text { Total number of } \\
\text { positions determined }\end{array}$} \\
\hline & Saastamoinen & NWP approach \\
\hline \multicolumn{3}{|l|}{ 5. Sept. 2000} \\
\hline $00: 00-06: 00$ & 13559 & 17116 \\
\hline 12:00-18:00 & 16007 & 17452 \\
\hline \multicolumn{3}{|l|}{ 14. Sept. 2000} \\
\hline $00: 00-06: 00$ & 17298 & 18181 \\
\hline $12: 00-18: 00$ & 13768 & 14286 \\
\hline
\end{tabular}

It is also interesting to investigate how the ambiguity resolution is affected by the different approaches for tropospheric corrections. Of the positions used for Table 2 and 3 the percentage of positions based on fixed ambiguities are shown in Table 4.

Table 4. Percentage fixed solutions

\begin{tabular}{|c|c|c|}
\hline \multirow[t]{2}{*}{$\begin{array}{l}\text { Kinematic } \\
\text { results }\end{array}$} & \multicolumn{2}{|c|}{$\begin{array}{l}\text { Percentage of positions based on } \\
\text { fixed ambiguities }\end{array}$} \\
\hline & Saastamoinen & NWP approach \\
\hline \multicolumn{3}{|l|}{ 5. Sept. 2000} \\
\hline $00: 00-06: 00$ & $84 \%$ & $98 \%$ \\
\hline $12: 00-18: 00$ & $99 \%$ & $100 \%$ \\
\hline \multicolumn{3}{|l|}{ 14. Sept. 2000} \\
\hline 00:00-06:00 & $99 \%$ & $100 \%$ \\
\hline $12: 00-18: 00$ & $100 \%$ & $100 \%$ \\
\hline
\end{tabular}

Generally the number of fixed ambiguities are correlated with the position accuracies as given in Table 2, where the statistics is based on both fixed and float solutions. As expected, with the relatively long baseline lengths processed in this case, some ambiguities were fixed to wrong integer values. Previous experience with the software has shown that it does have problems with solving ambiguities to the correct integer values, when processing kinematic data from longer baselines [6].

\section{Conclusion and future work}

A new approach for estimating the tropospheric delay based on numerical weather predictions has been described in this paper. Tests indicate that the accuracy of tropospheric zenith delays based on the DMI-HIRLAM-E data are better than the accuracy of the Saastamoinen global tropospheric delay model. The static and kinematic positioning tests also show that the method does have a potential for 
improving GPS positioning performance, and especially with the first two data sets there is a general improvement in position accuracy for both the static and kinematic results.

However, for the data collected during the period with high tropospheric activity, the positioning performance is generally not improved with the NWP approach. This is in contrast to what was expected, since a better modelling of the tropospheric delay was expected to be more beneficial during times with larger variability in the delay values. The explanation is assumed to be found in the processing software used for the tests. Using another test setup, where the tropospheric correction routine can be built directly into the source code of the programme, is therefore necessary in order to solve this problem. Also tests with more data, for instance from other climate regions, or from days with high ionospheric activity, are necessary to finally conclude whether the method is feasible or not.

The new technique is dependent on the accuracy of the weather models and tests should also be carried out using other weather models, for instance with a smaller or larger grid spacing, in order to investigate how this will affect the positioning results.

\section{Acknowledgements}

The Danish Meteorological Institute (DMI) is acknowledged for the DMI-HIRLAM-E data, and Henrik Vedel from DMI is thanked for advice and discussions related to the use of the data. The National Land Survey of Sweden is acknowledged for the SWEPOS GPS data, and the National Survey and Cadastre - Denmark (KMS) is acknowledged for the Danish GPS data. Bo Madsen from KMS is thanked for helping with the Bernese data processing.

\section{References}

1. Beutler, G., E. Brockmann, R. Dach, P. Fridez, W. Gurtner, U. Hugentobler, J. Johnson, L. Mervart, M. Rothacher, S. Schaer, T. Springer, R. Weber (2000). Bernese GPS Software. Astronomical Institute, University of Berne.

2. Bevis, M., S. Businger, S. Chriswell, T. A. Herring, R. A. Anthes, C. Rocken, R. H. Ware (1994). GPS Meteorology: Mapping Zenith Wet Delays onto
Precipitable Water. Journal of Applied Meteorology, 33: 379-386.

3. Brunner, F. K., M. Gu (1991). An improved model for the dual frequency ionospheric correction of GPS observations. Manuscripta Geodaetica, 16:205-214.

4. Cannon, M. E. (1997). Carrier Phase Kinematic Positioning: Fundamentals and Applications. In Geodetic Applications of GPS, Lecture Notes for Nordic Autumn School edited by Bo Jonsson. Number 16 in Reports in Geodesy and Geographical Information Systems, National Land Survey of Sweden.

5. Hopfield, H.S.(1969). Two-quartic Tropospheric Refractivity Profile for Correcting Satellite Data. Journal of Geophysical Research, 74(18): 4487-4499.

6. Jensen, A. B. O., M. E. Cannon (2000). Performance of Network RTK Using Fixed and Float Ambiguities. Proceedings of the 2000 National Technical Meeting of the Satellite Division of the Institute of Navigation (ION NTM 2000). Pages 797-805.

7. Jensen, A. B. O. (2002). Numerical Weather Predictions for Network RTK. Publication Series 4, volume 10. National Survey and Cadastre - Denmark.

8. Johansson, J. M. (1997), Modelling of the Earth Atmossphere in Space Geodetic Applications. In Geodetic Applications of GPS, Lecture Notes for Nordic Autumn School edited by Bo Jonsson. Number 16 in Reports in Geodesy and Geographical Information Systems, National Land Survey of Sweden.

9. Langley, R. (1996). Propagation of the GPS Signal. In Kleusberg, A. and P. J. G.Teunissen (eds) GPS for Geodesy, Lecture Notes in Earth Sciences. SpringerVerlag.

10. Mendes, V. B. (1999). Modelling the neutral-atmosphere propagation delay in radiometric space techniques. Ph.D. dissertation. Report number 199. Department of Geodesy and Geomatics Engineering, University of New Brunswick, Fredricton.

11. Niell, A. E. (1996). Global mapping functions for the atmosphere delay at radio wavelengths. Journal of Geophysical Research, 101(B2):3227-3246.

12. Pany, T., P. Pesec, G. Stangl, (2001). Atmospheric GPS Slant Path Delays and Ray Tracing Through Numerical Weather Models, a Comparison. Physics and Chemistry of the Earth. 26A(3):183-188.

13. Saastamoinen, J. (1973). Contributions to the Theory of Atmospheric Refraction. Bulletin Geodesique. Printed in three parts, 105:279-298, 106:383-397, 107:13-34.

14. Sass, B. H., N. W. Nielsen, J. U. Jørgensen, B. Amstrup, M. Kmit (2000). The Operational HIRLAM System at DMI. Scientific Report 00-26. Danish Meteorological Institute. Copenhagen

15. Schueler, T. (2001). On Ground-based GPS Tropospheric Delay Estimation. Ph.D. thesis, Universität der Bundeswehr, München.

16. Seeber, G. (1993). Satellite Geodesy. Foundations, Methods and Applications. Walter de Gruyter.

17. Vedel, H., K. S. Mogensen, X.-Y. Huang (2001). Calculation of zenith delays from meteorological data, comparison of NWP model, radiosonde and GPS delays. Physics and Chemistry of the Earth, 26A(6-8):497-502. 